\title{
PREFERENCIAS CONYUGALES EN TULUMBA (CÓRDOBA). COMIENZOS DE LOS SIGLOS XIX Y XX
}

\author{
MARITAL PREFERENCES IN TULUMBA (CÓRDOBA): BEGINNINGS OF \\ NINETEENTH AND TWENTIETH CENTURIES
}

\author{
Claudio Francisco Küffer*, Sonia Edith Colantonio
}

\begin{abstract}
*Centro de Investigaciones y Estudios sobre Cultura y Sociedad. Consejo Nacional de Investigaciones Científicas y Técnicas-Universidad Nacional de Córdoba (CONICET-UNC). Sede: UNCba. Córdoba. Argentina
\end{abstract}

\section{PALABRAS CLAVE biodemografía; isonimia; pares repetidos}

\begin{abstract}
RESUMEN Tulumba es una región del noroeste cordobés poco abordada desde la biodemografía. Un estudio previo señala variaciones en preferencias conyugales entre parientes y entre linajes, comparándose fines del siglo XVIII y comienzos del XIX. En este trabajo se propuso continuar ese análisis diacrónico. Las fuentes utilizadas fueron: para comienzos del siglo XIX (1806-1815) registros parroquiales de bautismos del curato de Tulumba, separados en "españoles" y "castas"; para la primera década del siglo XX (1901-1910) actas de nacimientos del Registro Civil y Capacidad de las Personas de Villa Tulumba. Se observó, mediante isonimia, una preferencia por el casamiento entre parientes notoriamente mayor en el siglo XX y, entre grupos del XIX, la mayor en españoles. Por su parte, en todos los casos se encontró una frecuencia
\end{abstract}

de casamientos entre linajes familiares mucho más elevada que la esperada, con alta o muy alta significación estadística, repitiéndose mayor preferencia en el siglo XX y, dentro del XIX, mayor en españoles que en castas. Se interpretaron estos resultados como consecuencia, en parte, de una estrategia patrimonial debida a las decrecientes oportunidades que brindaba la región en el siglo XX, respecto del XIX, sin descartar el posible efecto de preferencias conyugales por origen geográfico. En la convulsa época de comienzos del siglo XIX, la más notoria preferencia de los españoles por casarse con parientes o entre determinados linajes podría reflejar una forma de protección ante la inminencia de las luchas independentistas y guerras civiles. Rev Arg Antrop Biol 20(1), 2018. doi:10.17139/raab.2018.0020.01.03

\section{KEY WORDS biodemography; isonymy; repeated pairs}

ABSTRACT Tulumba is a region in the northwest of Córdoba province that has hardly been studied from a biodemographic viewpoint. Previous research shows variations in marital preferences between relatives and between lineages, comparing the late-eighteenth and early-nineteenth centuries. This work has sought to continue that diachronic analysis. The sources used were as follows: for the earlynineteenth century (1806-1815), baptismal registers belonging to the parish of Tulumba, separated into "Spanish" and "Mixed", while for the first decade of the twentieth century (1901-1910), birth certificates from the Registro Civil y Capacidad de las Personas de Villa Tulumba were surveyed. A significantly higher preference was observed, through isonymy, for marriage between relatives in the twentieth century; and, in the nineteenth-century groups, a higher preference among the Spanish. Meanwhile, in all cases,

El actual departamento Tulumba se encuentra en el norte de la provincia de Córdoba, como puede verse en Figura 1. Es uno de los menos poblados actualmente, contando junto con otras jurisdicciones del norte y oeste provinciales, con una menor industrialización y crecimiento vegetativo y más alta emigración que sus vecinas del centro y sudeste. Sin embargo, estas características de la región noroeste, y de Tulumba en particular, no siempre fueron tales.

La historiografía da cuenta de que, como the frequency of marriage among certain family lineages was higher than expected, with high or very high statistical significance, and again with a higher isonymic trend in the twentieth century; while, in the nineteenth century, it was also higher in the Spanish than in the Mixed group. These results were interpreted as arising in part from a strategy that could have been useful to preserve the patrimony, prompted by the declining opportunities the region presented in the twentieth century compared to the previous one, without ruling out the possible effect of marital preference driven by geographical origin. During the turbulent times of the earlynineteenth century, this more marked preference showed by the Spanish for marrying relatives or among certain lineages may reflect a form of protection, given the imminence of the struggle for Independence and the civil wars. Rev Arg Antrop Biol 20(1), 2018. doi:10.17139/raab.2018.0020.01.03

otras sociedades iberoamericanas, la cordobesa tardo-colonial (y hasta bien entrado el período

\footnotetext{
Financiamiento: CONICET, PIP 112-201101-00796; FONCyT, PICT 2014-1523.

*Correspondencia a: Claudio Francisco Küffer. Centro de Investigaciones y Estudios sobre Cultura y Sociedad (CONICET y UNC). Av. Ciudad de Valparaíso S/N. 5000 Córdoba. Argentina.E-mail: c.kr.005@gmail.com
}

Recibido 18 Octubre 2016; aceptado 29 Junio 2017

doi:10.17139/raab.2018.0020.01.03 


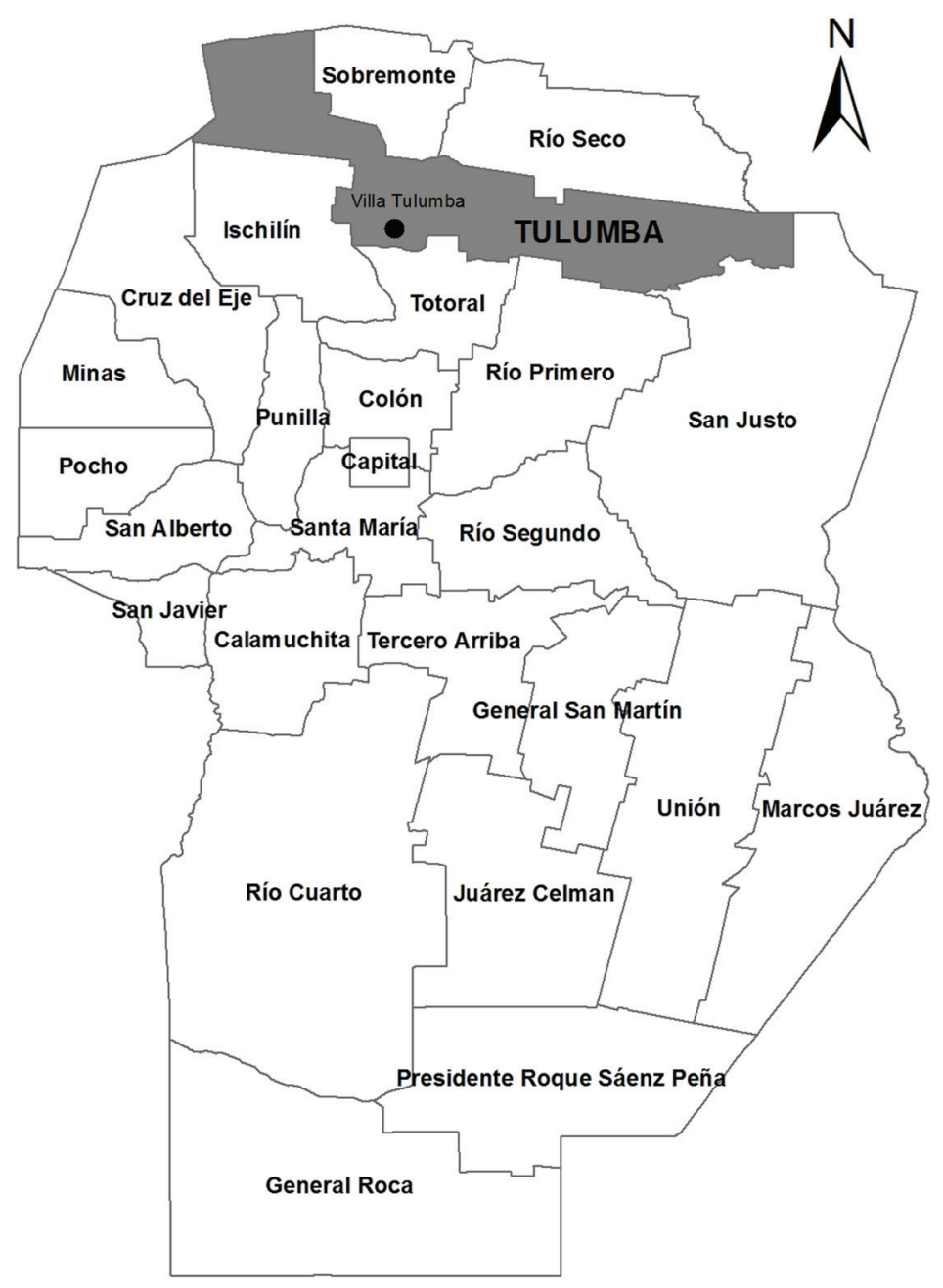

Fig. 1. Mapa de la provincia de Córdoba con su división departamental actual. Fuente: elaboración propia.

independiente) era estamental y multiétnica, con muy escasos matrimonios entre diferentes grupos socio-étnicos y una ilegitimidad elevada (Celton, 1997; Ferreyra, 1997). Por un lado se encontraban los "españoles", clase dominante conformada por españoles metropolitanos y europeos junto con sus descendientes y, por otro, "el resto", que suelen llamarse en conjunto "naturales" y agrupa a "negros", "indios" y los frutos del mestizaje entre estos dos grupos y con los españoles. Ya en el período estadístico en los censos de población no se incorporaron, hasta bien avanzado el siglo XX, preguntas referentes a dimensiones étnicas, en contraste con lo que venía ocurriendo anterior- mente (Otero, 2011). Sin embargo, tal proceder en los censos de 1869, 1895 y 1914, se debió tanto a dificultades técnicas en la de medición de la variable "color", de naturaleza gradual, como a la convicción de la futura desaparición del componente "no blanco" en la población, en parte por debilidades físicas constitutivas y en parte por el mestizaje con la "raza blanca" (Otero, 2011). Por tanto, los prejuicios basados en la filiación étnica no parecieron desaparecer con la abolición de la esclavitud, o con la sanción de la Constitución Nacional en 1853, sino que se tendió a una deliberada negación (al menos en términos del discurso oficial) de una sociedad multicultural. 
Para la Córdoba histórica existe una regionalización de la provincia en noroeste, centro $\mathrm{y}$ sudeste, que tiene en cuenta peculiaridades geo-ambientales, económicas y de poblamiento (Celton, 1993). Las dos primeras regiones son de poblamiento temprano, caracterizadas por sierras, valles serranos y piedemonte, mientras que el sudeste poblado más tardíamente en forma estable, es tierra de llanuras. Al menos hasta comienzos del siglo XIX la producción estaba más diversificada en la zona serrana, que concentraba la mayor proporción de la población provincial y tenía una más elevada densidad poblacional, contándose entre sus principales actividades la invernada de mulas y confecciones de algodón y lana (Tell, 2008).

El territorio de la actual provincia de Córdoba ocupaba un lugar central en el Virreinato del Río de la Plata, creado en 1776, y representaba un paso casi obligado para personas y mercancías desde y hacia Buenos Aires, Chile y el Alto Perú (Assadourian et al., 2005). Sin embargo, la puesta en vigencia del Reglamento de Libre Comercio entre España e Indias promulgado en 1778 benefició, en términos económicos, a la zona del litoral con la apertura del puerto de Buenos Aires, desplazando paulatinamente a la vía terrestre hacia el Alto Perú con el consiguiente perjuicio para el norte del país (Calvimonte, 1986). La desigualdad entre regiones se acrecentó en el siglo XIX, en el sentido de una clara preeminencia de Buenos Aires, seguido del Litoral, Cuyo y el interior (que incluía a Córdoba), si bien con variaciones intrarregionales y diferentes ritmos de estancamiento o despegue económicos (Gelman, 2011). Muy notorio fue el contraste entre Buenos Aires y Córdoba que, si bien presentó la economía más consolidada del interior durante el período colonial, pasó por un período complicado en las décadas post-independentistas, mientras que la economía porteña destacó sobre el resto cada vez con mayor diferencia (Gelman y Santilli, 2011). Con todo, tanto en lo económico cuanto en lo social el estado provincial favoreció la "continuidad colonial" (por caso, la citada regionalización con predominio de la región serrana), característica del perfil histórico cultural en la primera mitad del siglo XIX (Romano, 2002).

La situación de la provincia de Córdoba, con las diferencias regionales antes descrita, experimentó cambios importantes en el llamado "período de transición" que comenzó alrededor de 1870 y en el que coexistieron características coloniales con las predominantes en la "revolución agraria" (1895 hasta fines de siglo) y más aún en el "ciclo de expansión" (1900-1914), que tuvieron al ferrocarril como elemento dinamizador, con viraje del polo de atracción poblacional, tanto intra como interprovincial y extranjera, desde el noroeste hacia la región pampeana concordante con la expansión económica de este área (Moreyra, 1992). El ferrocarril favoreció el nacimiento y asentamiento de nuevas localidades, pero también dejó más aisladas a antiguas comunidades como la propia Villa de Tulumba (Calvimonte, 2002). Más concretamente, si bien las líneas férreas permitieron un notorio desarrollo comercial e industrial en la región pampeana, los departamentos del norte y oeste provincial tenían mucha menor extensión de vías férreas, y ni siquiera cruzaban por el territorio de todos los departamentos (Solveira, 1997a). Tal contraste puede observarse en Figura 2, donde puede apreciarse no solo el dominio del trazado este-oeste por sobre el norte-sur, sino que la diferencia en la trocha (ancho de la vía ferroviaria) de las líneas involucradas encarecían los trasbordos (Solveira, 1997b).

En suma, en el marco provincial se produjo un desplazamiento del polo productivo y comercial desde el centro-noroeste hacia el centro-sudeste, provocando un incremento de aislamiento poblacional y emigración en la región noroeste, que incluye a Tulumba. Seguramente las oscilaciones político-económicas condicionaron la conformación de estructuras poblacionales diferentes, ya que la zona del noroeste cordobés se ha mantenido relativamente aislada y aparentemente más libre de influencias foráneas que el centro y sur provinciales. Estas particularidades pueden tratarse desde una perspectiva biodemográfica.

La biodemografía es una disciplina integradora de componentes biológicos y socioculturales en las poblaciones humanas que, con base en datos demográficos, pretende delinear el tipo de estructura genética de las poblaciones, los mecanismos que actuaron en su conformación, y aquellos que podrían modificarla a futuro. Si bien Tulumba ha recibido 


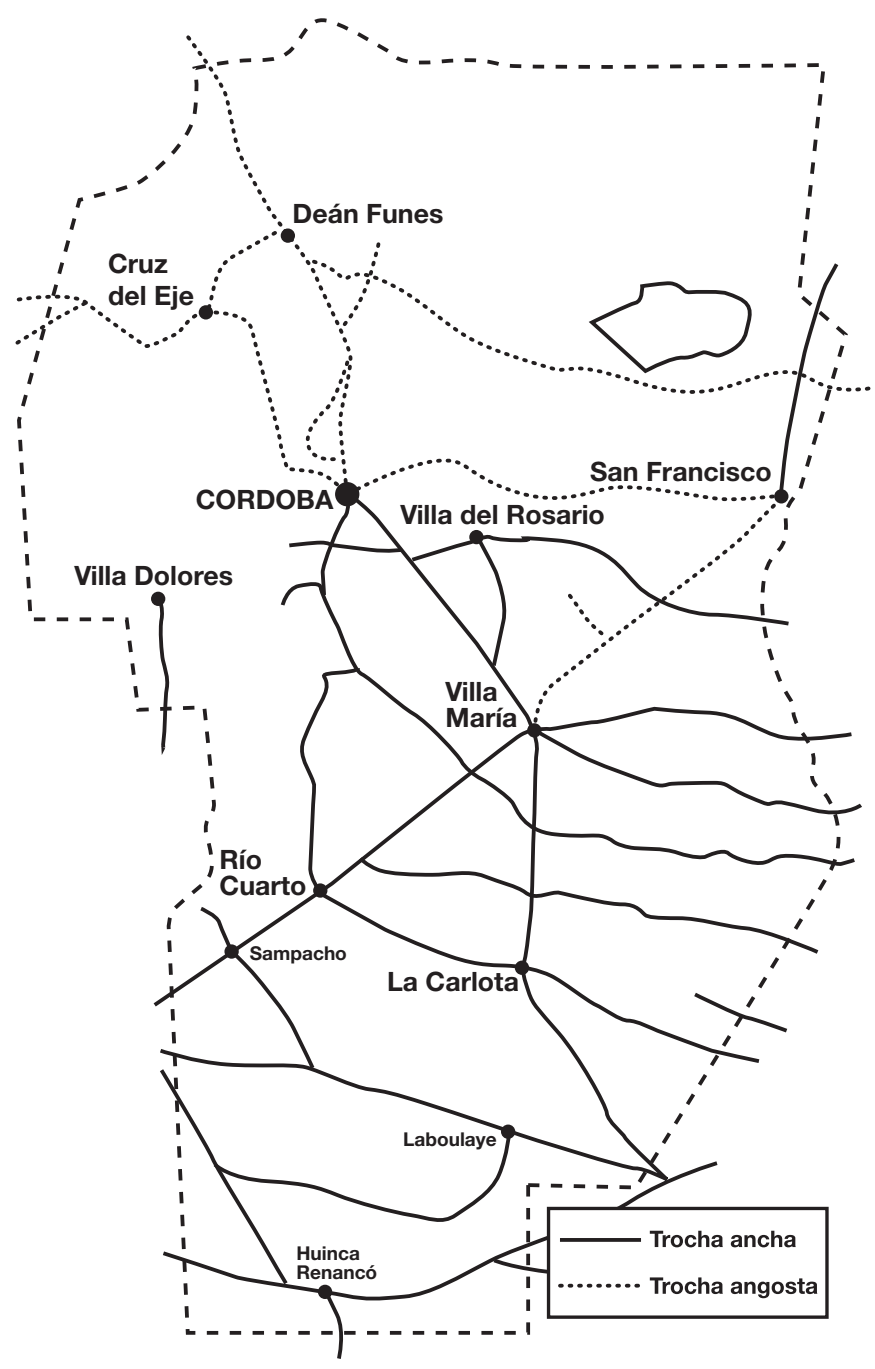

Fig. 2. Red ferroviaria en explotación, año 1918. Fuente: Archivo General de Gobierno, Ministerio de Obras Públicas, año 1920, tomo 3, fs. 269. Tomado de Solveira (1997b).

atención desde un enfoque socio-demográfico y para períodos históricos acotados (Celton et al., 2014; Küffer, 2016), no hubo un seguimiento longitudinal de ciertas variables a través de registros cronológicamente distantes. En un estudio biodemográfico previo que compara tres períodos de fines del siglo XVIII y comienzos del XIX, se encontraron variaciones en las preferencias conyugales que parecen coherentes con las disímiles situaciones socio-políticas que la población fue atravesando (Küffer et al., 2015). Sí hay estudios más abundantes desde la óptica biodemo- gráfica para otras poblaciones cercanas como las de los departamentos Pocho (Colantonio y Nordio, 1990; Colantonio y Celton, 1996) y Minas (López y Colantonio, 1999) para las que se señalan una importante emigración y son descriptas con características de semiaislados.

El objetivo de esta investigación fue continuar el análisis biodemográfico diacrónico de las preferencias matrimoniales en Tulumba, en términos de cónyuges emparentados y entre líneas familiares, relacionándolas con los cambios socio-políticos y económicos ocurridos, comparándose los comienzos de los siglos XIX 
y XX. Ello con base en la "isonimia" y "pares repetidos", métodos que se fundamentan en los apellidos presentes en las comunidades bajo estudio para estimar la consanguinidad y el parentesco.

La isonimia es un método desarrollado por Crow y Mange (1965) y modificado por Crow (1980), que se utiliza en estudios biodemográficos, especialmente útil si el acervo génico original no está presente (poblaciones históricas, por ejemplo). La consanguinidad puede estar influenciada por prácticas matrimoniales que incluyen elección o evitación de cónyuge emparentado. A partir de la isonimia, que considera parientes a los individuos portadores del mismo apellido (isónimos), se puede inferir la gravitación de estas prácticas contrastándose dos componentes de la consanguinidad total: la no aleatoria, que da cuenta de las citadas preferencia y evitación, y la aleatoria que está dada por la frecuencia de los diferentes apellidos en la población (de casados, si se habla de isonimia marital) y depende mayormente del tamaño de esta última. Lo importante de esta distinción estriba en que si bien la consanguinidad aleatoria y el tamaño poblacional tienden a presentar una correlación inversa, la no aleatoria no depende estrictamente de variables demográficas, sino que está influenciada por factores socio-culturales (Relethford y Jaquish, 1988).

Las poblaciones pueden presentar subdivisiones que respondan a múltiples causas, desde geográficas hasta estratificación social, que actuarían como barreras para la elección conyugal. Tal subdivisión puede estimarse a partir del método pares repetidos, que da cuenta de la frecuencia con que ciertos linajes se casan entre sí. El método fue desarrollado por Lasker y Kaplan (1985), y ampliado por Chakraborty (1985, 1986). Se denomina "pares repetidos" a la frecuencia con que cualquier par de apellidos que se da en un matrimonio se repite en otra pareja (Lasker, 1991).

Vale aclarar que en este trabajo se tuvieron en cuenta las uniones de derecho, vía eclesiástica o bien vía civil, mientras que la reproducción de buena parte de la población donde se produjo principalmente el mestizaje, se operó fuera del matrimonio, tanto en el siglo XIX como en el $\mathrm{XX}$.

\section{MATERIAL Y MÉTODOS}

\section{Fuentes}

Para comienzos del siglo XIX se tomaron los registros de bautismos de los años 1806 a 1815, copias de los cuales han sido microfilmadas por miembros de la Iglesia de Jesucristo de los Santos de los Últimos Días y cuyos originales se encuentran en el Archivo de la Catedral Nuestra Señora del Carmen, Prelatura de Deán Funes. En las partidas constan lugar y fecha, nombres y apellidos del bautizado y de los padres, de los padrinos, de quien concretó la ceremonia y edad de la criatura. Son comunes los casos en que sólo figura uno de los progenitores, casi siempre la madre, y no son infrecuentes los "huérfanos de padre y madre". Los bautismos están separados en dos libros. Uno corresponde a los "españoles" y otro a los "naturales". Asimismo, para conocer el total poblacional se tuvo en cuenta el censo civil de 1813 para el curato de Tulumba, que abarca a los actuales territorios de Tulumba y Totoral separados recién en 1858 (Calvimonte, 2002). Dicho censo puede consultarse en el Archivo Histórico de la Provincia de Córdoba y fue abordado biodemográficamente en varias oportunidades (ver por ejemplo: Colantonio et al., 2007; Colantonio et al., 2013).

Para el inicio del siglo XX se relevaron las actas de nacimientos entre 1901 y 1910, consultadas en el Registro Civil y Capacidad de las Personas de Villa Tulumba, Córdoba. En ellas se consignan lugar y fecha del nacimiento, lugar de residencia del declarante, nombres y apellidos del nacido, los padres (y su nacionalidad) abuelos y testigos. En cuanto al total poblacional, se tuvieron en cuenta los brindados por los censos civiles de 1895 y 1914 puesto que, a diferencia del período anterior, no hubo un registro censal que cayera justo dentro del período analizado. Los originales de ambos se encuentran en el Archivo General de la Nación y copias digitalizadas de los mismos se consultaron para este trabajo en la Dirección General de Estadísticas y Censos del Gobierno de Córdoba (DGEyC, 2016).

Las fuentes descriptas para el siglo XIX se encuentran entre las correspondientes al período pre-estadístico, caracterizadas por algunas falencias importantes como son el porcentaje 
de los recuentos respecto del total poblacional real, la filiación etno-social y la edad, que parecieron fluctuar mucho conforme al criterio del empadronador y la persona relevada (Arretx et al.,1983; Ghirardi, 2007). Las tomadas para el siglo XX, en cambio, se encuadran en el período estadístico cuyos registros suelen estar mejor organizados (Arretx et al., 1983). Otra característica que los diferencia es que los primeros son eclesiásticos y los segundos son civiles, respondiendo de este modo a distintas instituciones (Iglesia y Estado) y realizados con diferentes propósitos. Con todo, las discrepancias entre las fuentes no fueron impedimento para realizar comparaciones entre ellas. Los responsables de relevar los datos parecieron tener el suficiente cuidado como para que sus anotaciones resultaran fiables, al menos en cuanto a la información requerida para esta investigación. El haber tomado los bautismos para el siglo XIX y los nacimientos para el XX obedeció principalmente a que durante el primer período todos los nacidos fueron bautizados, mientras que para el segundo no necesariamente todo nacido lo fue.

\section{Métodos}

En primer lugar se cotejaron los totales poblacionales y por sexo de los censos de 1813 , 1895 y 1913, para tener una aproximación al crecimiento vegetativo o la emigración/inmigración, que pudo haber tenido lugar; siempre teniendo en cuenta que el primero de los censos abarcaba a los actuales Tulumba y Totoral y los segundos sólo a Tulumba. Asimismo, se tuvo presente la proporción de extranjeros en cada uno de los totales poblacionales para saber si la inmigración pudo jugar un rol importante en las estimaciones aportadas por los métodos descritos a continuación. Para este último punto no se pudo tener en cuenta la inmigración desde otras provincias y menos aún desde la misma provincia, porque el dato no aparece especificado con tal detalle en los censos del siglo XX, al menos en sus conteos finales.

Se seleccionaron, de las partidas de bautismos y actas de nacimientos, los matrimonios en que el apellido de ambos cónyuges constaba en la partida o acta.

Por lo que se mencionó antes, que a comienzos del siglo XIX el curato de Tulumba no se correspondía exactamente con el departamento de principios del siglo XX del mismo nombre, se descartaron las partidas de 1806-1815 no realizadas en territorio del futuro departamento Tulumba (por ejemplo: bautizado en la Capilla Totoral); ello para poder comparar confiablemente lo acontecido en ambos períodos. Otra cuestión para tener en cuenta en las partidas del siglo XIX es que, si bien la denominación "naturales" es la utilizada en los registros para referirse a los no españoles, aquí se usó el término "castas" para englobar a los individuos producto del mestizaje entre los grupos de origen europeo, aborigen y africano. Se supuso que los anotados como "naturales" eran integrantes de las castas salvo que mediara alguna aclaración; se prefirió castas, no sólo por su uso común en la historiografía, sino porque "natural" era también empleado con otras connotaciones, como en "hijo natural", "natural de (un lugar)", e incluso a veces, en especial al referirse a Pueblos de indios, como sinónimo de aborigen. Se descartaron los matrimonios de clase dudosa (por figurar unas veces en el libro de españoles y otras en el de naturales), los conformados por esposos de diferente grupo socio-étnico o, en el caso de castas, si al menos un cónyuge era de condición esclava. Puesto que los análisis se basaron en el método de isonimia, las estimaciones se consideraron fiables para los españoles, sin presuponerse su fiabilidad para las castas, a las que se incluyó más a título comparativo. Por otra parte, se tuvieron en cuenta sólo las castas libres asumiéndose que una vez adquiridos los apellidos su transmisión fue más regular, semejando la de los españoles. Los esclavos, heredando el apellido de su eventual dueño y no el de su padre, no cumplían el principio de transmisión patrilineal.

Para los matrimonios de ambos períodos, 1806-1815 y 1901-1910, se calcularon los estimadores de consanguinidad a partir de la isonimia conyugal definidos por Crow y Mange (1965) y Crow (1980): consanguinidad aleatoria $\left(\mathrm{F}_{\mathrm{r}}\right)$ que, no aleatoria $\left(\mathrm{F}_{\mathrm{n}}\right)$ y total $\left(\mathrm{F}_{\mathrm{t}}\right)$. También se efectuó el cálculo del parentesco intra-poblacional $\left(\mathrm{R}_{\mathrm{iij}}\right)$, por el método de Lasker (1985).

La subdivisión poblacional se estimó mediante pares repetidos (RP), método ideado por Lasker y Kaplan (1985). Cabe recordar que "pares repetidos" alude a la frecuencia con que cualquier par de apellidos dado en un matrimo- 
nio se repite en otra pareja (Lasker, 1991). Por su parte, el valor de pares repetidos aleatorios $\left(\mathrm{RP}_{\mathrm{r}}\right)$ se obtuvo por la fórmula publicada por Chakraborty $(1985,1986)$. El indicador (RP$\left.\mathrm{RP}_{\mathrm{r}}\right) / \mathrm{RP}_{\mathrm{r}}$ se empleó para ponderar el valor observado respecto del esperado (Relethford, 1992). Para poner a prueba la hipótesis $R P=R P$, se utilizó el estadístico $z$, de acuerdo con Relethford (1992).

Se tuvo en cuenta el dato de origen geográfico (argentino/extranjero) por su posible influencia en las elecciones conyugales. Esto pudo hacerse para el segundo período, dado que en el primero esta información está ausente. Conjuntamente con ello, para tener un indicio de movimientos migratorios, se calculó el porcentaje de apellidos que estuvieron presentes entre los casados de principios del siglo XX que no lo estuvieron en el período anterior y viceversa.

Cabe recordar que se tuvieron en cuenta las uniones de derecho. De modo que lo esbozado a modo de conclusión se refiere a este tipo de uniones, sin poder extenderse a la población en general. Conforme con ello adicionalmente, se ponderó la relación entre hijos conyugales y nacidos fuera de matrimonio.

El armado de bases digitalizadas y los procedimientos estadísticos se realizaron mediante los programas Excel 2007 y SPSS 21.0, los correspondientes a isonimia y pares repetidos con programas elaborados $a d$ hoc y la Figura 1 fue graficada con el programa ArcMap 10.3

\section{RESULTADOS}

Los totales censales de la población y desagregados por sexo, junto con los de inmigrantes no argentinos, pueden verse en Tabla 1. Como puede apreciarse, hubo un claro aumento poblacional entre 1813 y 1895 , pero más acotado en 1914. Asimismo, en los tres registros las mujeres están más representadas. La inmigración aumentó claramente de un registro a otro, siendo en los tres casos los migrantes mayoritariamente varones.

Respecto de los hijos nacidos fuera del matrimonio, para el primer período (1806-1815), de un total de 1038 partidas los hijos no conyugales suman $349(33,62 \%)$, mientras que en el segundo (1901-1910) que cuenta con 2417 actas tienen una menor incidencia, $624(25,82 \%)$, sin dejar por ello de representar alrededor de $1 / 4$ de los nacimientos.

En Tabla 2 se muestran los totales de matrimonios de ambos períodos tenidos en cuenta para este trabajo.

En Tabla 3 se muestran los estimadores de consanguinidad y parentesco a partir de isonimia. Se puede observar la mayor relevancia del componente no aleatorio $\left(\mathrm{F}_{\mathrm{n}}\right)$, o preferencial, a comienzos del siglo $\mathrm{XX}$, valor que reflejaría un casamiento entre parientes más frecuente que en el siglo anterior, el que incluso es nulo en las

TABLA 1. Población total y por sexo en 1813, 1895 y 1914

\begin{tabular}{cccc}
\hline Censo & Varones & Mujeres & Total \\
\hline \multirow{2}{*}{1813} & 2364 & 2717 & 5081 \\
& $17(0,72)$ & $2(0,074)$ & $19(0,37)$ \\
\cline { 2 - 4 } 1895 & 5355 & 6032 & 11387 \\
& $84(1,57)$ & $29(0,48)$ & $113(0,99)$ \\
\cline { 2 - 4 } 1914 & 5972 & 7140 & 13112 \\
& $325(5,44)$ & $117(1,64)$ & $442(3,37)$ \\
\hline
\end{tabular}

En cursiva inmigrantes no argentinos y entre paréntesis su representatividad porcentual en el total.

TABLA 2. Parejas casadas para los períodos 18061815 y $1901-1910$

\begin{tabular}{cccc}
\hline Período & Origen & $\mathrm{N}$ & Total \\
\hline \multirow{2}{*}{$1806-1815$} & $\begin{array}{c}\text { Españoles } \\
\text { Castas }\end{array}$ & 221 & 352 \\
\cline { 2 - 4 } 1901-1910 & $\begin{array}{c}\text { Población } \\
\text { general }\end{array}$ & & 842 \\
\hline
\end{tabular}

TABLA 3. Estimaciones de los componentes de consanguinidad y parentesco intrapoblacional a partir de isonimia

\begin{tabular}{cccc}
\hline Estimador & \multicolumn{2}{c}{$1806-1815$} & $1901-1910$ \\
& Españoles & Castas & \\
\hline $\mathrm{F}_{\mathrm{r}}$ & 0,00383387 & 0,00391877 & 0,00234074 \\
$\mathrm{~F}_{\mathrm{n}}$ & 0,00070178 & $-0,00204239$ & 0,00962587 \\
$\mathrm{~F}_{\mathrm{t}}$ & 0,00453296 & 0,00188439 & 0,01194408 \\
$\mathrm{R}_{\mathrm{ii}}$ & 0,00766774 & 0,00783754 & 0,00468148 \\
\hline
\end{tabular}


castas. Dicho componente resultó en una mayor consanguinidad total $\left(\mathrm{F}_{\mathrm{t}}\right)$. El parentesco intrapoblacional $\left(\mathrm{R}_{\mathrm{ii}}\right)$, dado por una acrecentada variabilidad de apellidos, fue mínimo en el siglo XX.

En Tabla 4 pueden verse los guarismos de pares repetidos, cociente $\left(\mathrm{RP}-\mathrm{RP}_{\mathrm{r}}\right) / \mathrm{RP}_{\mathrm{r}} \mathrm{y}$ valor de $z$. Las preferencias entre líneas familiares en niveles estadísticamente significativos se dieron en ambos períodos $\mathrm{y}$, en el siglo XIX, en ambos grupos y con más notoriedad en españoles.

Finalmente, para ponderar el peso de la migración en la población general, se encontró que de las 842 parejas casadas en el período 19011910 sólo en 20 de ellas (2,38\%) tienen al menos un cónyuge de origen extranjero, generalmente el varón, y de esas 20 sólo 6 ( $0,71 \%$ del total $)$ cuentan con ambos esposos extranjeros, siempre de la misma nacionalidad. A su vez, comparando los apellidos de ambos períodos, se encontró que a comienzos del siglo XX 49,84\% de los apellidos no se encontraba presente en el siglo XIX. Por contraparte, de los apellidos encontrados en el XIX, el $22,22 \%$ no se encontraron en el XX.

TABLA 4. Pares repetidos, $\left(R P-R P_{r}\right) / R P_{r}$ y valor de $z$

\begin{tabular}{clll}
\hline \multirow{2}{*}{ Estimador } & \multicolumn{2}{c}{$1806-1815$} & $1901-1910$ \\
& \multicolumn{1}{c}{ Españoles } & \multicolumn{1}{c}{ Castas } \\
\cline { 2 - 4 } $\mathrm{RP}$ & 0,0006170 & 0,0003523 & 0,0003108 \\
$\mathrm{RP}_{\mathrm{r}}$ & 0,0002516 & 0,0002165 & 0,0001011 \\
$(\mathrm{RP}-\mathrm{RPr}) / \mathrm{RPr}$ & 1,45 & 0,63 & 2,07 \\
$\mathrm{z}=(\mathrm{RP}-\mathrm{RPr}) /$ & $9,27 * * *$ & $2,17^{* *}$ & $20,16^{* * *}$ \\
$\mathrm{ES}(\mathrm{RPr})$ & & & \\
\hline
\end{tabular}

$* * * p<0,001 ; * * p<0,01 ; * p<0,05$

\section{DISCUSIÓN}

Como se observó, la inmigración aumentó claramente de un registro a otro, y esto pudo haber jugado un papel importante en el incremento poblacional. De todos modos conviene resaltar que, al no contarse con el dato de la inmigración de corta distancia, la gravitación de esta variable demográfica en el incremento de efectivos puede ser engañosa. En efecto, si se tiene en cuenta el total de habitantes de origen foráneo a la provincia de Córdoba en 1813 (no mostrado en Tabla 1) el número total se eleva a 80 , representando el 1,57\% del total. Del mismo modo, podría ocurrir que las mujeres migraran principalmente desde cortas distancias y así, su movilidad quedara enmascarada en gran parte. Esto último se ha registrado en trabajos anteriores para poblaciones históricas cordobesas (ver, por ejemplo, Küffer y Colantonio, 2005). Con todo, habida cuenta del desbalance de sexos a favor de las mujeres, es lícito pensar que también hayan sido los varones quienes más emigraban. Dado que a partir de la información utilizada no pudo saberse si en los censos del siglo XX el comportamiento migratorio de la población fue o no similar al de comienzos del siglo XIX, los guarismos de la inmigración desde otros países pueden resultar de utilidad para contrastarlos con las estimaciones de consanguinidad y parentesco, pero siempre teniendo en cuenta la salvedad explicitada.

Antes de entrar en las elecciones conyugales en sí, conviene repetir que las estimaciones obtenidas se refieren al comportamiento de las parejas casadas, es decir a una parte de la población, y no puede ser extensible sin más a las elecciones de parejas por fuera del matrimonio. Ello habida cuenta de la importante ilegitimidad encontrada en los registros de ambos períodos.

Claramente, hubo en la población de tiempos más recientes mayor incidencia de casamientos entre parientes (dado el $F_{n}$ mayor considerablemente más elevado). Una explicación para esto es que el viraje del polo productivo del noroeste al sudeste provincial, mediado por la industrialización, fue dejando más aislada a la región. Concretamente, la antes mencionada creación del ferrocarril pudo actuar más que como comunicante, incluso entre comunidades cercanas, como aislante y empeorando la situación cuando perdió vigencia en la zona (Calvimonte, 2002). Demográficamente, pese a que la población provincial creció en forma sostenida, Tulumba como el norte cordobés en general, inició su declinación a pasos acelerados desde 1895, notándose claramente en el censo de 1980 (Calvimonte, 2002). El número de efectivos, ya entrado el siglo XXI resultó similar e incluso menor a los encontrados a comienzos del siglo XX (DGEyC, 2016). Estrategias económicas para una zona empobrecida respecto de sus contrapartes del centro y sudeste provin- 
cial, podrían explicar el aumento del casamiento entre isónimos. Tampoco puede descartarse, si bien los datos no permiten ir más allá de lo especulativo, que una mayor laxitud en las costumbres (recuérdese que al menos oficialmente, la Iglesia tuvo cada vez menor injerencia) permitió consagrar uniones fruto de cuestiones afectivas entre parientes, otrora mal vistas o incluso prohibidas. Lo antedicho se condice con el mínimo parentesco intrapoblacional $\left(\mathrm{R}_{\mathrm{ii}}\right)$ en el siglo $\mathrm{XX}$, dado por una acrecentada variabilidad de apellidos en dicho siglo.

Lo antedicho brindaría mayor respaldo a una intencionalidad en el casamiento entre parientes, no por falta de potenciales cónyuges no emparentados, sino como forma de paliar épocas difíciles que se venían sobrellevando.

Cerrando lo anterior, en el siglo XIX los españoles manifestaron mayores preferencias por parientes y esto podría interpretarse principalmente como forma de protección ante las luchas independentistas y luego las guerras civiles y sus posibles consecuencias. En efecto, es esperable que ante tales cambios quienes tenían más que perder cuidaran más su patrimonio mediante uniones consanguíneas.

Lo obtenido a partir de pares repetidos parece respaldar, en general, lo observado dentro de cada línea familiar. La significación más elevada en el siglo XX, seguida de españoles del siglo XIX y castas de ese mismo siglo, avala este supuesto. En otras palabras, el patrón de conyugalidad entre familias repite lo obtenido dentro de cada familia. Las posibles causas parecen las mismas: estrategias para salvaguardar lo patrimonial a comienzos del XIX y eso mismo sumado a un mayor aislamiento de la región (limitando la posibilidad de elegir cónyuges).

Los porcentajes de extranjeros en ambos períodos (Tabla 1) podrían sugerir una mayor incidencia de potenciales cónyuges "foráneos" en tiempos más recientes entre la población general. Sin embargo, es pertinente recordar que ese dato da cuenta de la presencia de extranjeros, no de los migrantes de menores distancias. Y caben destacarse dos aspectos: la información censal revisada no explicita el estado civil de esos extranjeros, de modo que podría tratarse de solteros pero también en muchos casos de familias o al menos matrimonios, ya constituidos. Por otra parte pudo haber existido cierta evitación de los locales por casarse con foráneos, junto con una preferencia de los propios extranjeros por cónyuges de su lugar de origen. Un fenómeno de este tipo se hizo patente, por ejemplo, para dos localidades del departamento San Justo (Córdoba) a fines del siglo XIX y comienzos del XX (Rios Villamil et al., 2015). De haber ocurrido también eso en Tulumba de comienzos del siglo $\mathrm{XX}$, pudo haber sido un componente no menor en la importante subdivisión poblacional, que quedó reflejada por el elevado valor $z$ en este siglo. Los matrimonios entre no locales o entre un/a local y un/a extranjera/o, no parecen suficientes por su escaso número, para dar cuenta de lo antes comentado. Sin embargo, tales matrimonios sólo dan cuenta de lo que ocurría en el momento del registro. En el período intermedio entre los registros pareció haberse operado una importante movilidad, tanto desde como hacia el área en estudio si bien no es posible afirmar, contando solo con los datos descriptos, si esa movilidad fue mayormente de corta o larga distancia. Pero sí es otro de los posibles factores que convendría agregar para explicar las acrecentadas preferencias por cónyuges emparentados y entre linajes. Es decir, que se casaran en elevada proporción entre cónyuges de similar origen geográfico, no necesariamente el de ellos mismos, sino el de sus padres o abuelos. Esto parece contradecir el creciente aislamiento del noroeste cordobés, si bien en forma paulatina durante el período considerado, citado por datos historiográficos, y reforzar la importancia de los apellidos como complemento de los datos de origen geográfico, en especial cuando estos son escasos o poco específicos (ver por ejemplo Küffer y Colantonio, 2005; Colantonio y Fuster, 2009).

Las tendencias observadas, al compararse españoles y castas del primer período, concuerdan con lo encontrado en un trabajo anterior (Küffer et al., 2015), si bien allí se trató de un período más acotado.

A título comparativo, en un estudio longitudinal que abarca más años que el presente, realizado para el departamento Pocho, del noroeste cordobés, entre 1810 y 1980 a partir principalmente de censos civiles (Colantonio y Nordio, 1990) se observaron hacia fines del período: merma en el crecimiento vegetativo, emigración elevada y muy baja inmigración, uno de cuyos 
indicadores es que sólo una pequeña proporción de la población tiene apellidos que no hayan estado presentes un siglo y medio atrás, junto con un fuerte incremento en el número de personas con los mismos apellidos, indicativo esto último de una elevada consanguinidad. Es posible que Tulumba haya experimentado algo semejante hacia la misma época, pero parece arriesgado afirmar que fue de tal magnitud. Para ello sería de utilidad la incorporación de una secuencia de años de finales del siglo XX, lo cual se plantea a futuro.

\section{CONCLUSIONES}

A comienzos del siglo XX la sociedad se mostró más proclive a la elección conyugal entre parientes, posiblemente como estrategia patrimonial debido a las decrecientes oportunidades que brindaba la región. El intercambio entre determinados linajes, estadísticamente significativo en los dos períodos y más aún en el del siglo XX, parecen avalar esta explicación. Sumado a que, al compararse para el XIX españoles y castas, los primeros exhibieron mayores preferencias por casamiento entre parientes y entre linajes. En suma, los vaivenes económicos parecieron jugar un rol de primer orden a la hora de contraer matrimonio. Pero ello sin descartar, para el segundo período, posibles preferencias entre cónyuges de similar origen geográfico.

\section{AGRADECIMIENTOS}

Se agradece muy especialmente a los organismos que financiaron este trabajo.

\section{LITERATURA CITADA}

Arretx C, Mellafe R, Somoza J. 1983. Demografía Histórica en América Latina. Fuentes y métodos. San José de Costa Rica: Centro Latinoamericano de Demografía.

Assadourian CS, Beato G, Chiaramonte, JC. 2005. Historia argentina. Tomo II. De la conquista a la independencia. Buenos Aires: Editorial Paidós.

Calvimonte LQ. 1986. Tulumba. Aspectos históricos de la villa y su contorno. Córdoba: El Copista.

Calvimonte LQ. 2002. Tulumba. Su historia civil y eclesiástica. Córdoba: BR Copias.

Celton DE. 1993. La población de la Provincia de Córdoba a fines del siglo XVIII. Buenos Aires: Academia Nacional de la Historia.

Celton DE. 1997. Selección matrimonial y mestizaje en Córdoba. En: III Jornadas de Historia de Córdoba. Córdoba: Junta Provincial de Historia de Córdoba. p 327-344.

Celton DE, Küffer C, Colantonio SE. 2014. Control político, “desorden” social. Tulumba, en la campaña cordobesa, entre dos siglos. En: Ghirardi MM, editora. Territorios de lo cotidiano. Del antiguo Virreinato del Perú a la Argentina Contemporánea. Rosario: Prohistoria. p 153163.

Chakraborty R. 1985. A note on the calculation of random RP and its sampling Variance. Hum Biol 57:713-717.

Chakraborty R. 1986. Erratum. Hum Biol 58:991.

Colantonio SE, Celton DE. 1996. Estructura de una población semiaislada actual: reproducción, selección natural y deriva genética. Rev Esp Antrop Biol 17:105-127.

Colantonio SE, Nordio OR. 1990. Identificación de una población semi-aislada en la provincia de Córdoba. Córdoba: Publicaciones del Instituto de Estadística y Demografía, Facultad de Ciencias Económicas, Universidad Nacional de Córdoba.

Colantonio SE, Fuster V. 2009. Migration of ethnic groups within Cordoba province (Argentina) during political crises following the Colonial period. Popul Rev 48:103118. DOI: 10.1353 /prv.0.0018

Colantonio SE, Fuster V, Küffer C. 2007. Isonymous structure in the white population of Córdoba, Argentina, in 1813. Hum Biol 79:491-500. doi: 10.1353/ hub. 2008.0002

Colantonio SE, Fuster V, Román-Busto J. 2013. Poblaciones y parentescos. Relaciones biológicas evidenciadas a través de los apellidos. En: Colantonio SE, editora. Población y Sociedad en tiempos de lucha por la emancipación. Córdoba: CIECS (CONICET-UNC). p 499-523.

Crow JF. 1980. The estimation of inbreeding from isonymy. Hum Biol 52:1-14.

Crow JF, Mange AP. 1965. Measurement of inbreeding from the frequency of marriages between persons of the same surname. Eugen Quart 12:199-203. doi:10.1080/19485 565.1965 .9987630

DGEyC (2016) Dirección General de Estadísticas y Censos. Secretaría General de la Gobernación. Gobierno de la Provincia de Córdoba. Disponible en: http://estadistica. cba.gov.ar/

Ferreyra MC. 1997. El matrimonio de las castas en Córdoba. 1700-1779. En: Actas III Jornadas de Historia de Córdoba. Córdoba: Junta Provincial de Historia de Córdoba. p 285-326.

Gelman J. 2011. Introducción. Desequilibrios regionales, desigualdades sociales. Las economías argentinas en el siglo XIX. En: Gelman J, coordinador. El mapa de la desigualdad en la Argentina del siglo XIX. Rosario: Prohistoria Editores. p 11-45.

Gelman J, Santilli D. 2011. Crecimiento económico, desigualdad regional y distribución de la riqueza. Córdoba y Buenos Aires después de la independencia. En: Gelman J, coordinador. El mapa de la desigualdad en la Argentina del siglo XIX. Rosario: Prohistoria Editores. p 71-94.

Ghirardi MM. 2007. Fuentes para estudios de población en la etapa pre-estadística. En: Torrado S, compiladora. Población y bienestar en la Argentina del primero al segundo centenario. Una historia social del siglo XX. Tomo I. Buenos Aires: Edhasa. p 309-336.

Küffer C. 2016. Diferenciación social y mestizaje en Tulumba (Córdoba) a partir de Bautismos de fines del siglo XVIII y comienzos del XIX. En: Siegrist N, Olivero Guidobono S, Barreto Messano I, coordinadoras. Atravesando barreras: movilidad socio-étnica y cultural en Hispano América, siglos XVII-XIX. Sevilla: Egregius Ediciones. p 118-135.

Küffer C, Colantonio SE. 2005. Migración en la ciudad de Córdoba en el primer tercio del siglo XIX a partir de 
los datos del Censo de 1832 (apellidos y origen geográfico). Rev Arg Antrop Biol 7:79-93. doi:10.17139/ raab.2005.007.2.\%25g

Küffer C, Colantonio SE, Celton DE. 2015. Características biodemográficas en la población del curato de Tulumba (Córdoba) a fines del siglo XVIII y comienzos del XIX. Rev Arg Antrop Biol 17:1-12. doi:10.17139/ raab.2015.0017.02.08

López AM, Colantonio SE. 1999. Evolución del comportamiento reproductivo y estructura poblacional en el Dpto. Minas (Prov.de Córdoba, 1980-1991). Rev Arg Antrop Biol 2:41-54. doi:10.17139/raab.1999.002.1.\%25g

Lasker GW. 1985. Surnames and genetic structure. Cambridge: Cambridge University Press. doi:10.1017/ cbo9780511983351

Lasker GW. 1991. Revisión: datos sobre los apellidos hispanoamericanos en los estudios de la biología humana. An Antrop 28:107-128.

Lasker GW, Kaplan BA. 1985. Surnames and genetic structure: repetition of the same pairs of names of married couples, a measure of subdivision of the population. Hum Biol 57:431-440.

Moreyra BI. 1992. Crecimiento demográfico y expansión económica en el espacio pampeano cordobés durante el modelo primario-exportador (1880-1930). Córdoba: Centro de Estudios Históricos.
Otero H. 2011. El concepto de población en el sistema estadístico de Argentina, 1869-2001. Estatística e sociedade $1: 7-25$.

Relethford JH. 1992. Analysis of marital structure in Massachusetts using repeating pairs of surnames. Hum Biol 64:25-33.

Relethford JH, Jaquish CE. 1988. Isonymy, inbreeding, and demographic variation in historical Massachusetts. Am J Phys Anthropol 77:243-252.

Rios Villamil A, Colantonio SE, Mangeaud A. 2015. Population Structure in Rural Communities of Córdoba, Argentina: Marriage Patterns and Immigration Effect during Early 20th Century. International Journal of Social Science Studies 3:87-93. doi:10.11114/ijsss.v3i6.1128

Romano S. 2002. Economía, sociedad y poder en Córdoba. Primera mitad del siglo XIX. Córdoba: Ferreyra Editor.

Solveira BR. 1997a. Los ferrocarriles del norte y oeste de Córdoba, proyectos y realizaciones. En: Actas III Jornadas de Historia de Córdoba. Córdoba: Junta Provincial de Historia de Córdoba. p. 139-159.

Solveira BR. 1997b. Las inversiones en ferrocarriles, 18701920. En: Moreyra BI, Solveira BR, compiladoras. Estado, economía y sociedad en Córdoba, 1880-1950, I. Córdoba: Centro de Estudios Históricos. p. 69-121.

Tell S. 2008. Córdoba rural, una sociedad campesina (17501850). Buenos Aires: Prometeo Libros. 